\title{
Trickle Flow Liquid-Solid Mass Transfer and Wetting Efficiency in Small Diameter Columns
}

\author{
Rita Joubert and Willie Nicol* \\ Department Chemical Engineering, University of Pretoria, Pretoria, South Africa
}

\begin{abstract}
A novel method to simultaneously measure liquid-solid-mass-transfer and external wetting efficiency was employed at column to particle ratios of 10,6 and 3 . Two prewetting procedures representing the upper (Kan) and lower (Levec) hysteresis branches were used. For a multipoint distributor on a Kan-prewetted bed wetting efficiency and the specific masstransfer coefficient were almost unaffected by column diameter. The multipoint distributor on a Levec-prewetted bed exhibited a decrease in specific mass transfer with decreasing column diameter. Pointsource experiments resulted in significantly lower wetting and mass-transfer measurements with an increasing trend with respect to decreasing column diameter. The results indicate that with proper distribution and prewetting, the effect of column diameter on averaged wetting and liquid-solid mass transfer is almost negligible, a powerful result considering the importance of these parameters on reaction experiments.
\end{abstract}

Keywords: Specific liquid-solid mass transfer, Wetting efficiency, Column to particle ratio

\section{Introduction}

Laboratory scale representation of commercial trickle bed reactors has is a type of experiment employed by numerous research institutions. Whether the study entails a new reaction process or an existing one, the aim is usually to be representative using the smallest amount of catalyst. When the catalyst test work is of a conceptual nature catalyst fines can be used order to obtain an initial estimate. This approach will however not suffice if a more accurate prediction/representation is required. The first step in improving the mimicking properties of the laboratory scale reactor is usually to use the same catalyst pellets as that of the commercial unit. With similar packing one would ideally like to recreate the hydrodynamic properties of the commercial unit. This will only be possible if the superficial gas and liquid 
velocities of the commercial unit are used in the laboratory setup but given the extended length of commercial reactors it is not always possible to represent the complete commercial reactor in a single laboratory run. Numerous investigators opt for lower superficial velocities in the laboratory units in order to operate at the same space velocity as that of the commercial reactor. In the case of a trickle bed reactor where gas and liquid moves downward through the bed, lower velocities will entail poorer catalyst wetting and external mass transport (gas to liquid and liquid to solid), it might also cause a regime shift from the higher to the lower interaction regime. Although the latter will defect the mimicking of the small scale experiment, the most likely poorer performance of the laboratory unit will at least be 'forgiving' when scaling to larger units (at higher velocities). This said, knowledge on the wetting and external transport properties of the system will assist in improved up scaling and understanding.

In order to keep the catalyst amount and feed requirements of the laboratory reactor low, the diameter should be as small as possible. It will be preferable to rather have a long bed with a small diameter to keep the superficial velocities at realistic values. The trade-off in doing this lies in the contribution of wall effects for smaller diameter columns, where the contribution of the higher porosity near-wall zone affects the overall hydrodynamic properties of the column (Templeman and Porter, 1965). Numerous studies have been performed on this specific topic and a list is supplied in table 1 . The column to particle diameter ratio $(\mathrm{D} / \mathrm{dp})$ is commonly used to define the critical point where the wall effects are negligible and values between 8 and 20 are suggested in literature (see table 1). Different criteria were used in determining these values, with wall flow quantification via annular collectors, pressure drop and holdup measurements and radial porosity quantification being the most prominent. Not to be confused with the minimum $\mathrm{D} / \mathrm{dp}$ value for hydrodynamic consistency is the maximum $\mathrm{D} / \mathrm{dp}$ value for negligible radial temperature profiles in the case where heat generation due to reaction is a concern (Mears, 1971).

When considering a trickle bed reactor operating in the absence of radial temperature profiles, the most crucial hydrodynamic parameters affecting the reaction outcome are the external mass transfer steps (gas-liquid and liquid solid) and the catalyst wetting. Compared to liquid holdup and pressure drop these parameters are more directly linked to the overall reaction rate. It will therefore be interesting to know the effect of these overall parameters on $\mathrm{D} / \mathrm{dp}$ in order to extend the criteria for deciding upon a minimum value, specifically when 
considering laboratory scale reaction studies. Since numerous commercial trickle bed reactors are liquid reagent limited and near isothermal, Liquid Solid Mass Transfer (LSMT) and catalyst external wetting was chosen as hydrodynamic parameters in this study. A novel electrochemical method (Joubert and Nicol, 2011) able to simultaneously measure LSMT and wetting efficiency was employed in three different columns with respective $\mathrm{D} / \mathrm{dp}$ ratios of 3,6 and 10. Multipoint distributors (MPD) as well as point source distributors (PSD) were used at the upper and lower bound of the hysteresis envelope.

Table 1: Some minimum column to particle diameter ratios from literature

\begin{tabular}{|c|c|c|c|}
\hline Reference & Min D/dp ratio & Method & Criteria \\
\hline Baker et al. (1935) & $>8$ & Radial porosity distribution & Constant average bed porosity \\
\hline $\begin{array}{l}\text { Benenati and Brosilow } \\
\text { (1962) }\end{array}$ & $>10$ & Radial porosity distribution & Porosity variation damped out \\
\hline $\begin{array}{l}\text { Tempelman and Porter } \\
\text { (1965) }\end{array}$ & $>8$ & Annular collectors & Constant wall flow \\
\hline $\begin{array}{l}\text { Mehta and Hawley } \\
\text { (1969) }\end{array}$ & $>7$ & Pressure drop & $\begin{array}{l}\text { Bed pressure drop equal to } \\
\text { pressure drop calculated using } \\
\text { Ergun equation }\end{array}$ \\
\hline Gianetto et al. (1970) & $>13$ & Absorption & $\begin{array}{l}\text { Constant gas-liquid interfacial } \\
\text { area }\end{array}$ \\
\hline Gierman (1988) & $>16$ & Annular collectors & Uniform liquid distribution \\
\hline Larachi et al. (1991) & $>11.5$ & Hold-up and pressure drop & $\begin{array}{l}\text { Consant average liquid } \\
\text { distribution and constant } \\
\text { pressure drop }\end{array}$ \\
\hline $\begin{array}{l}\text { Al-Dahhan and } \\
\text { Dudukovic (1994) }\end{array}$ & $>20$ & $\begin{array}{l}\text { Pressure drop and Liquid } \\
\text { hold up }\end{array}$ & Uniform liquid distribution \\
\hline Attou and Boyer (1999) & $>8$ & Radial porosity distribution & Constant average bed porosity \\
\hline Sie and Krishna (1998) & $>20$ & Radial porosity distribution & Constant average bed porosity \\
\hline Raichura (1999) & $>16$ & Pressure drop & $\begin{array}{l}\text { Constant average bed porosity } \\
\text { and constant pressure drop }\end{array}$ \\
\hline $\begin{array}{l}\text { Winterberg and Tsotsas } \\
(2000)\end{array}$ & $>10$ & Modelling & $\begin{array}{l}\text { Bed pressure drop equal to } \\
\text { pressure drop calculated using } \\
\text { Ergun equation }\end{array}$ \\
\hline De Klerk (2003) & $>12$ & Radial porosity distribution & $\begin{array}{l}\text { Constant average bed porosity } \\
\text { and constant pressure drop }\end{array}$ \\
\hline Ren et al. (2005) & $>10$ & NMR & $\begin{array}{l}\text { Amount of liquid flow at wall } \\
\text { not higher than liquid flow in } \\
\text { centre region }\end{array}$ \\
\hline
\end{tabular}




\section{Experimental}

A diagram of the experimental setup is shown in figure 1. The setup consists of three clear PVC columns with internal diameter of $12 \mathrm{~mm}, 26 \mathrm{~mm}$ and $44 \mathrm{~mm}$, and a length of $1 \mathrm{~m}$. The columns are operated with an atmospheric outlet and at room temperature (approximately $298 \mathrm{~K}$ ). The liquid (a solution containing $1 \mathrm{M} \mathrm{NaOH}, 0.02 \mathrm{M} \mathrm{K}_{4} \mathrm{Fe}(\mathrm{CN})_{6}, 0.003 \mathrm{M} \mathrm{K}_{3} \mathrm{Fe}(\mathrm{CN})_{6}$ ) and gas phase $\left(\mathrm{N}_{2}\right)$ is fed concurrently to the bed. Two types of distributors were used in this study. One system employs a Point source (PSD) with a hole diameter of $1 \mathrm{~mm}$. In the other system the liquid enters through a needle distributor, MPD, containing 9, 36 and 59 needles (corresponding to the $12 \mathrm{~mm}, 26 \mathrm{~mm}$ and $44 \mathrm{~mm}$ columns) with an internal diameter of $1 \mathrm{~mm}$ in a square pitch arrangement. In both systems nitrogen enters the column through a $12 \mathrm{~mm}$ id pipe positioned below the liquid distributor plate.

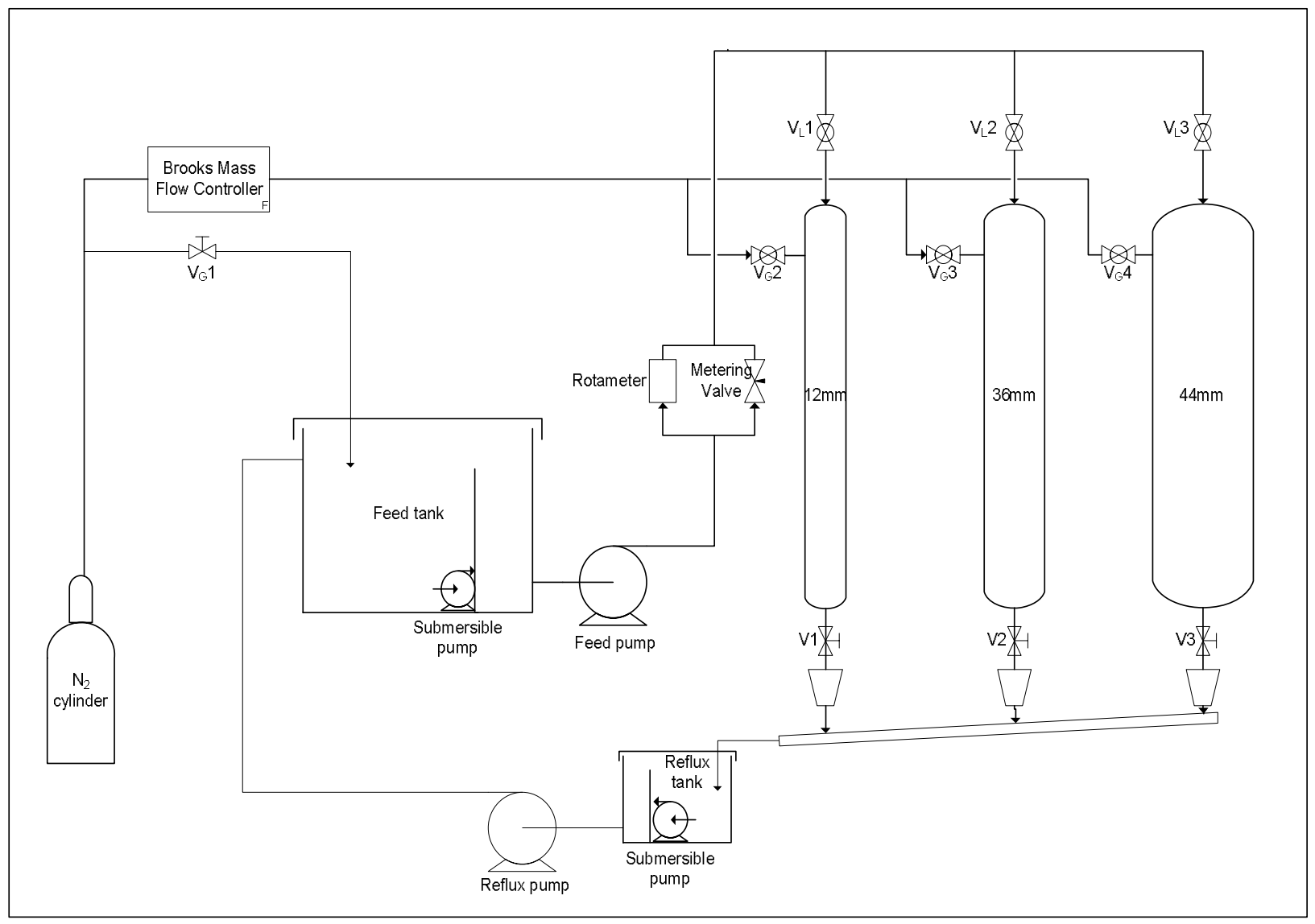

Figure 1: Experimental set-up

The electrolyte was kept in a nitrogen atmosphere to eliminate the possibility of degradation. The physico-chemical properties of the electrolyte are given in table 2. Multiple nickel coated 
beads $(4.5 \mathrm{~mm})$ in close contact were used as the rate-limiting cathode. Different cathodes were inserted at two locations within the bed (shown in figure 2), separated from one another by $4 \mathrm{~mm}$ glass beads. A large section of nickel beads (4-6 times the size of the largest cathode) at the outlet of the column acted as the anode. The reagent concentration for the anode reaction is ten times that needed for the cathode reaction to ensure rate limitation at the cathode. The standard electrochemical method described by Rao and Drinkenburg (1985) is used to measure the liquid-solid mass transfer at the surface of the electrodes. The current through the system, for an applied potential difference of $1000 \mathrm{mV}$, is measured using a NI PXI-4071 Digital Multimeter with 7 1/2 digit accuracy supplied by NI-Solutions. Liquid-solid mass transfer coefficients are calculated using the measured current as shown in equation 1.

$I_{l i m}=\mathrm{nCFA} k_{l s}$

equation 1

Table 2: Physico-chemical properties of the electrolytic solution

\begin{tabular}{|l|l|l|}
\hline Property & Value & Units \\
\hline Density & 1020 & $\mathrm{~kg} / \mathrm{m}^{3}$ \\
\hline Viscosity & 0.0019 & Poise \\
\hline Surface tension & 29.5 & Dynes $/ \mathrm{cm}$ \\
\hline Diffusion coefficient & $6.49 \times 10^{-9}$ & $\mathrm{~m}^{2} / \mathrm{s}$ \\
\hline
\end{tabular}

Two preconditioning methods were used in order to investigate the range of possible wetting efficiencies and mass transfer coefficients. The first method, referred to as the Kan mode, represents the upper hydrodynamic bound. This is achieved by operating the column in the high interaction, or pulsing, regime for a couple of minutes before switching the liquid and gas flow rates to the desired operating velocity. The second method represents the lower hydrodynamic bound for preconditioned beds and will be referred to as the Levec mode. In this mode the column pre-wetting is achieved by flooding the column with liquid. The column is then allowed to drain under gravity for 20 minutes, after which the liquid and gas phases is introduced to the column at the desired operating flows. (Loudon et al., 2006) 
The wetting efficiency was measured by operating the electrochemical circuit in the kinetic regime, where the intrinsic reaction rate of the electrochemical reaction controls the current. This is achieved when low voltages are applied between the electrodes (Joubert and Nicol, 2011). Unlike the mass transfer controlled regime where the current does not alter with an increase in applied voltage, the kinetic regime is characterised by a linear relationship between current and applied voltage (Hanratty and Campbell, 1983). This regime behaves similar to a liquid phase chemical reaction operating in the absence of external (liquid-solid) mass transfer effects, where the superficial velocity in the bed has no influence on the reaction rate. The effect of superficial velocity under liquid flooded conditions ( $100 \%$ surface wetting) was used to establish the voltage required where mass transfer effects are negligible. At $50 \mathrm{mV}$ it was observed that a the current reading was similar for superficial velocities of 1 and $20 \mathrm{~mm} / \mathrm{s}$ and accordingly a voltage of $20 \mathrm{mV}$ was used for all the wetting efficiency measurements.

The measured current at $20 \mathrm{mV}$ is directly proportional to the area available for electrochemical reaction. In order to calibrate this relationship composite anodes of different sizes where packed for the calibration run (figure 2(a)). The external cathode areas varied from $0.000636 \mathrm{~m}^{2}$ (10 beads) to $0.0 .1272 \mathrm{~m}^{2}$ (2000 beads), by packing different size electrodes in each of the three columns. Figure 3 shows the current measurements of the minimum and maximum velocities. The non-zero offset of the total current measurement can be attributed to the contribution of the non-faradaic current (current produced by physicochemical processes other than the redox reaction). The non-faradaic current was measured by repeating the calibration experiments with a $\mathrm{NaOH}$ only solution. The faradaic current (caused by electrochemical reaction) can be obtained by the difference between the two linear regressions (figure 3) and behaves according to the expected outcome. It is important to note that the influence of non-faradaic current in the mass transfer measurements is negligible and therefore not reported (Wang, 2006). 


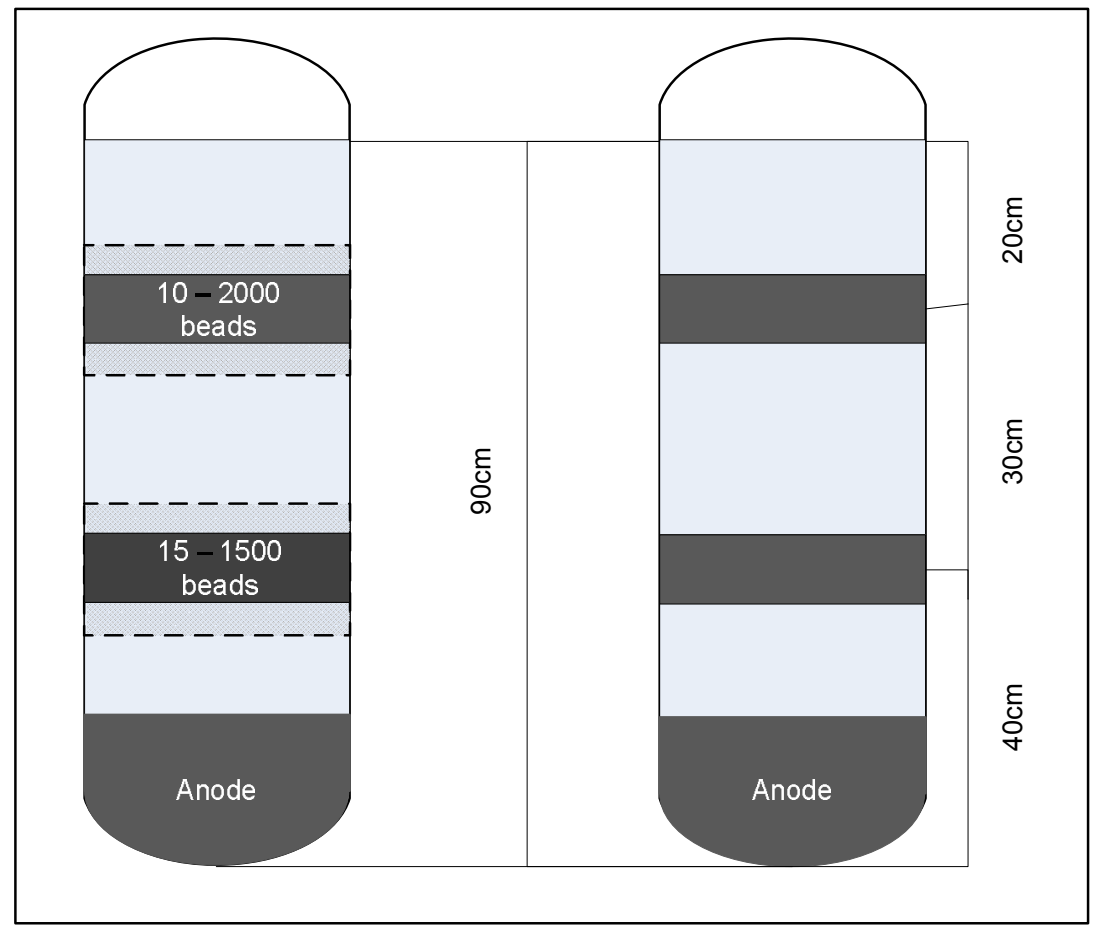

(a)

(b)

Figure 2: Electrode placement (a)calibration experiments-flooded flow only (b)wetting efficiency and liquid-solid mass transfer experiments

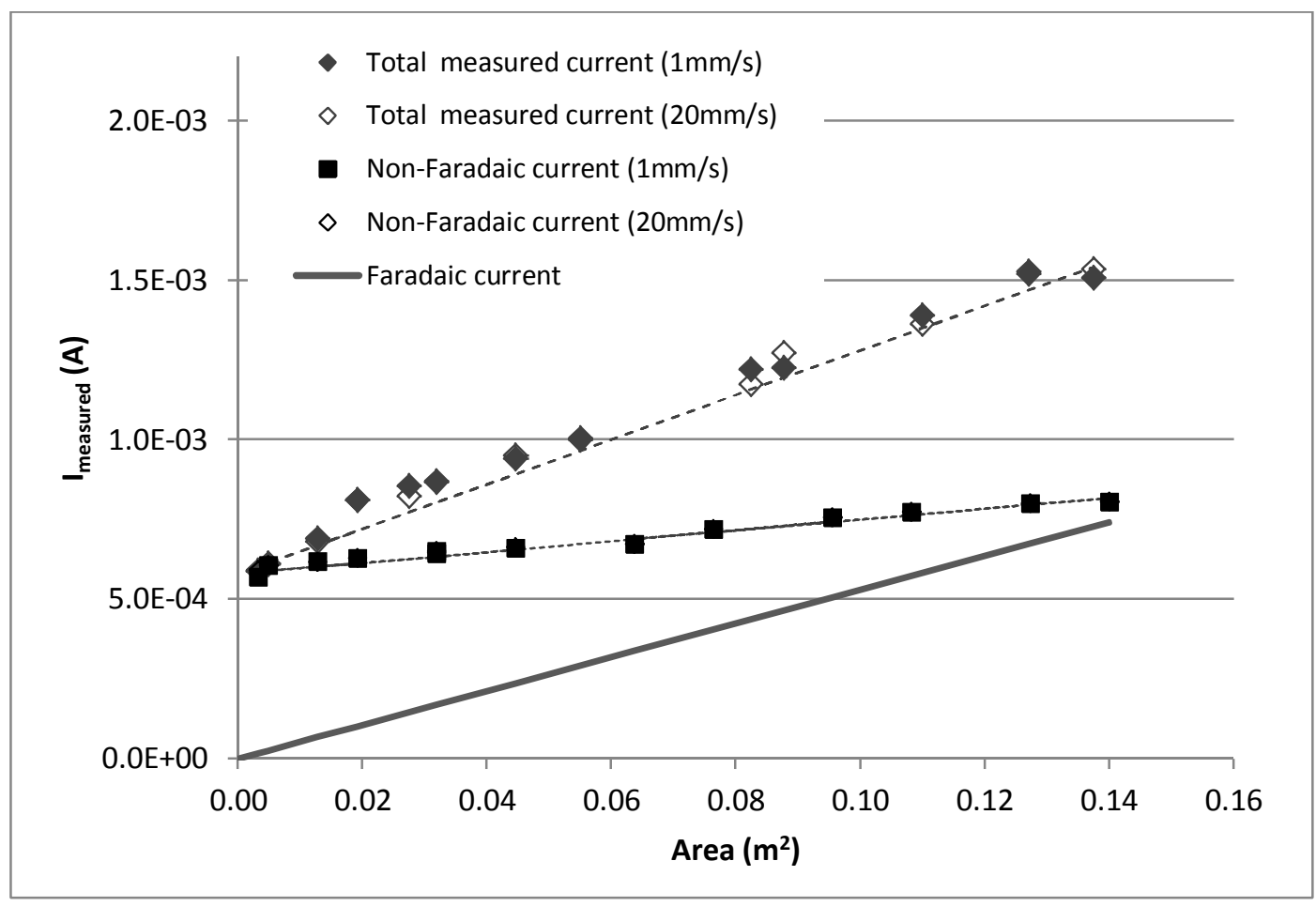

Figure 3:Calibration curve of measured current and cathode area 


\section{Results and Discussion}

All reported data points represent the average from 3 readings obtained, with the prewetting procedure performed between the acquisitions. Although not indicated on the graphs, the repeatability of all reported points was within $4 \%$ absolute deviation from the mean. All the data is represented in figure 4 and figure 5, while figure 6 provides an alternative representation of the top cathode at a liquid velocity of $2 \mathrm{~mm} / \mathrm{s}$. It is important to note that the LSMT measurements supplied in figure 5 gives the value of the wetted area transfer coefficient and that the overall LSMT measurement was divided by the wetting efficiency (figure 4) to obtain the coefficient value.

The first striking result is the limited amount of wetting variation observed for the different column sizes when a MPD was used. The slightly lower values for the Levec prewetted beds are to be expected and are in agreement to the findings of van Houwelingen et al. (2006). Although not reported in this paper, the wetting readings were also similar to readings obtained from a $68 \mathrm{~mm}$ column $(\mathrm{D} / \mathrm{dp}=15)$ by Joubert and Nicol (2011) where the exact method was employed. The reading represents a radial average and does not distinguish between wall and centre wetting (attempts at annular and core electrode packing were unsuccessful). Whether a wetting gradient exist and what the gradient will be remains unknown, although calometric analysis performed by Baussaron et al.(2007) exhibited no radial wetting variation for beds with proper distribution (multiple needles). In contrast, the point source results (PSD) show a clear dependency with respect to column diameter where the wetting decreases as the column diameter increase. This is to be expected as the liquid flow for the larger column requires more bed depth to properly distribute up to the walls. Variation with regards to bed depth indicates that wetting is improving down the bed, but not to the extent that the wetting values of the MPD is obtained. The column length to diameter aspect ratio for the bottom electrode in the small column is 42 , and even at this relative depth the PSD wetting measurements is far lower than that of the MPD.

The second striking result is the close proximity of the Kan-mode specific LSMT measurements for different column sizes employing a MPD. Similar to the wetting results these measurements correspond to the $\mathrm{D} / \mathrm{dp}=15$ measurements (Joubert and Nicol, 2011) The $1 \mathrm{~mm} / \mathrm{s}$ readings have a peculiar deviation from the observed trend that corresponds to the 
findings of van der Merwe and Nicol (2005) where a critical velocity of $2 \mathrm{~mm} / \mathrm{s}$ was defined below which poor utilization of the bed volume occurred. The Levec-mode MPD measurements differ, apart from being lower in general there is a decrease in kls for the smallest column. A major bed depth variation for kls is also observed for the Levec-mode MPD results. With regards to the PSD measurements the LSMT coefficients are much lower than the corresponding MPD readings, with a decreasing trend with respect to an increase in diameter. Since the amount of wetted area has no influence on kls, the surface specific mass transfer appears to be much lower that of the MPD experiments. A plausible explanation for the severely low values lies in the existence of isolated liquid globules in the bed. The trapped globules or pockets formed during prewetting but did not receive irrigation afterwards. The extend of anion $\mathrm{Fe}(\mathrm{CN})_{6}{ }^{2-}$ conversion on the cathode is extremely low and accordingly a globule will give a stable wetting reading for prolonged periods of time. The mass transfer readings on the globule surfaces will however be very low due to the stagnant nature of the liquid and accordingly the effective mass transfer area is over predicted by the values in figure 4 . The lower kls value obtained for the Levec-mode MPD might be linked to the same argument, but it is also possible that lower average velocities near the solid surface (compared to stagnant conditions) caused the differences.

Accent should be place on the Kan-mode MPD results. The major finding of this work is that proper prewetting and distribution will ensure that the average LSMT and wetting characteristic of small diameter columns (as low as $\mathrm{D} / \mathrm{dp}=3$ ) are similar to those of larger columns. Despite the differences in pressure drop, radial porosity and liquid distribution for these small columns, the primary rate determining hydrodynamic parameters (LSMT and external wetting) seems to be unaffected. This suggests that near isothermal reaction experiments under mass transfer limitation will be unaffected by the column diameter up to a minimum of 3 column diameters or even less. It will definitely be interesting to test the postulate under real reaction conditions. 
Levec

a)

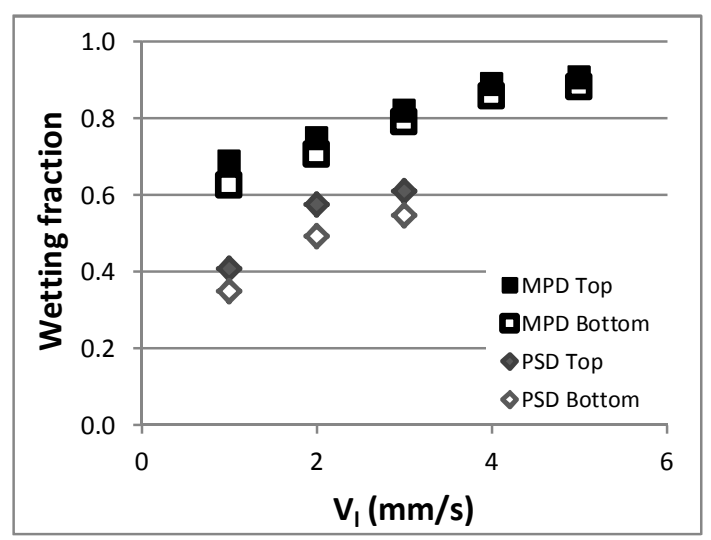

b)

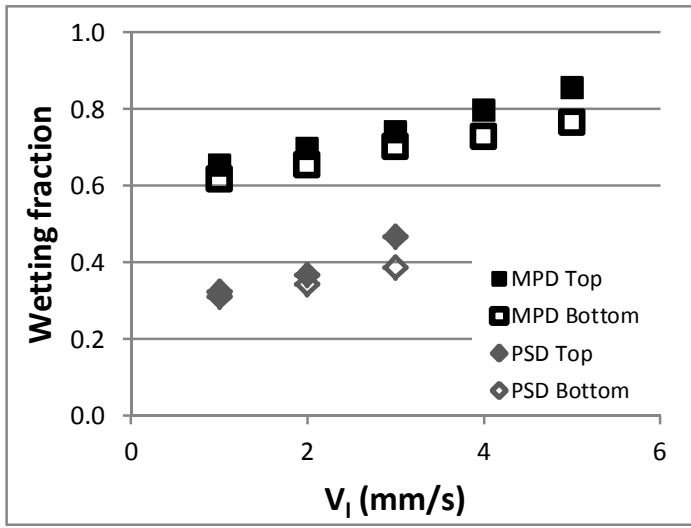

c)

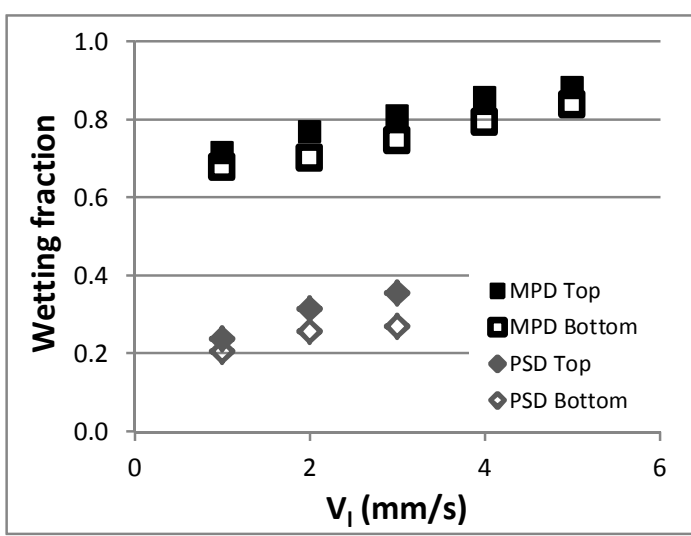

Kan
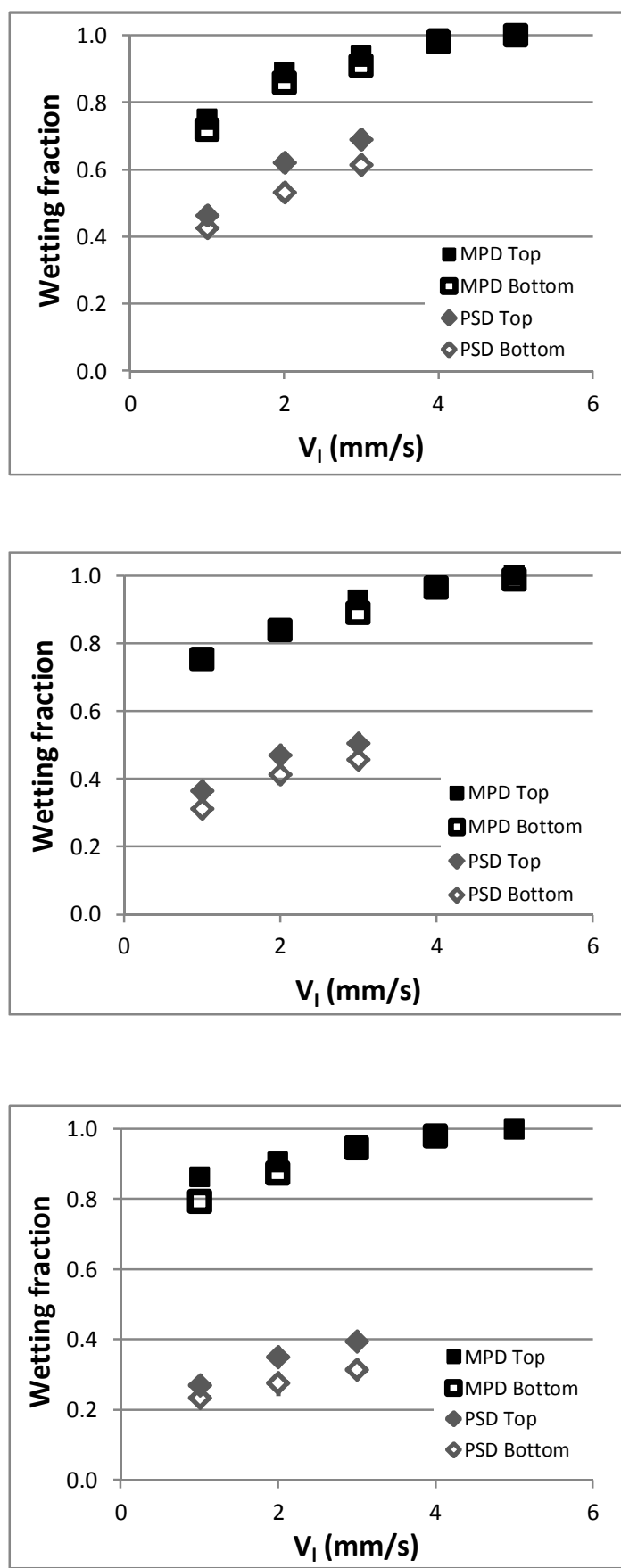

Figure 4: Wetting efficiency using a MPD and a PSD $\left(V_{g}=20 \mathrm{~mm} / \mathrm{s}\right)$ a)D/dp=3 b)D/dp=6 c)D/dp=10 
Levec

a)

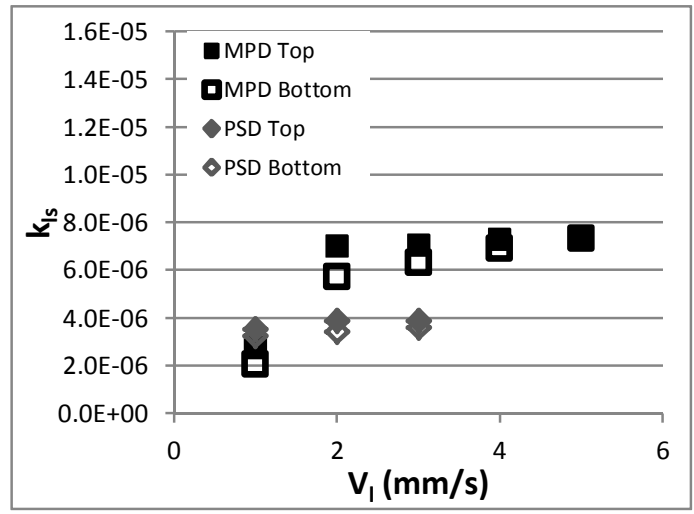

b)

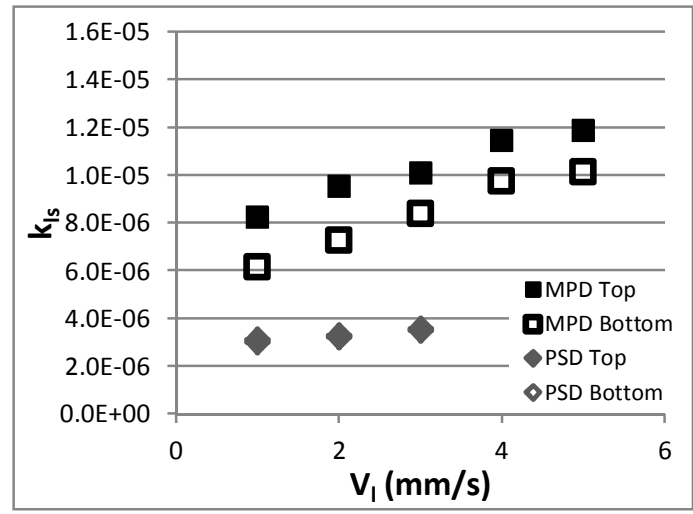

c)

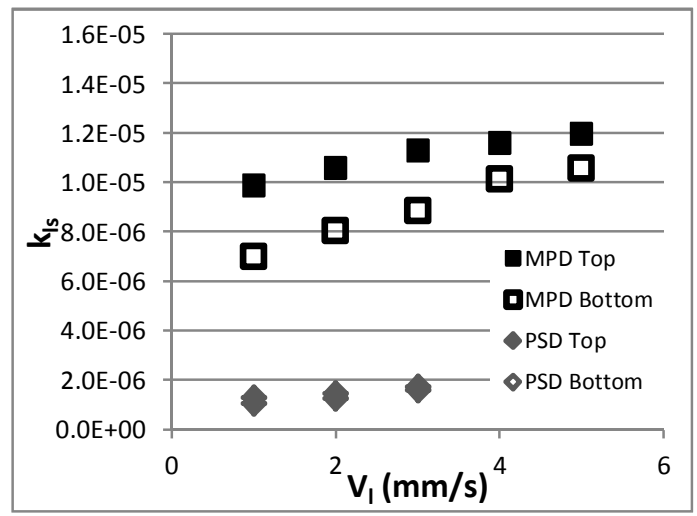

Kan
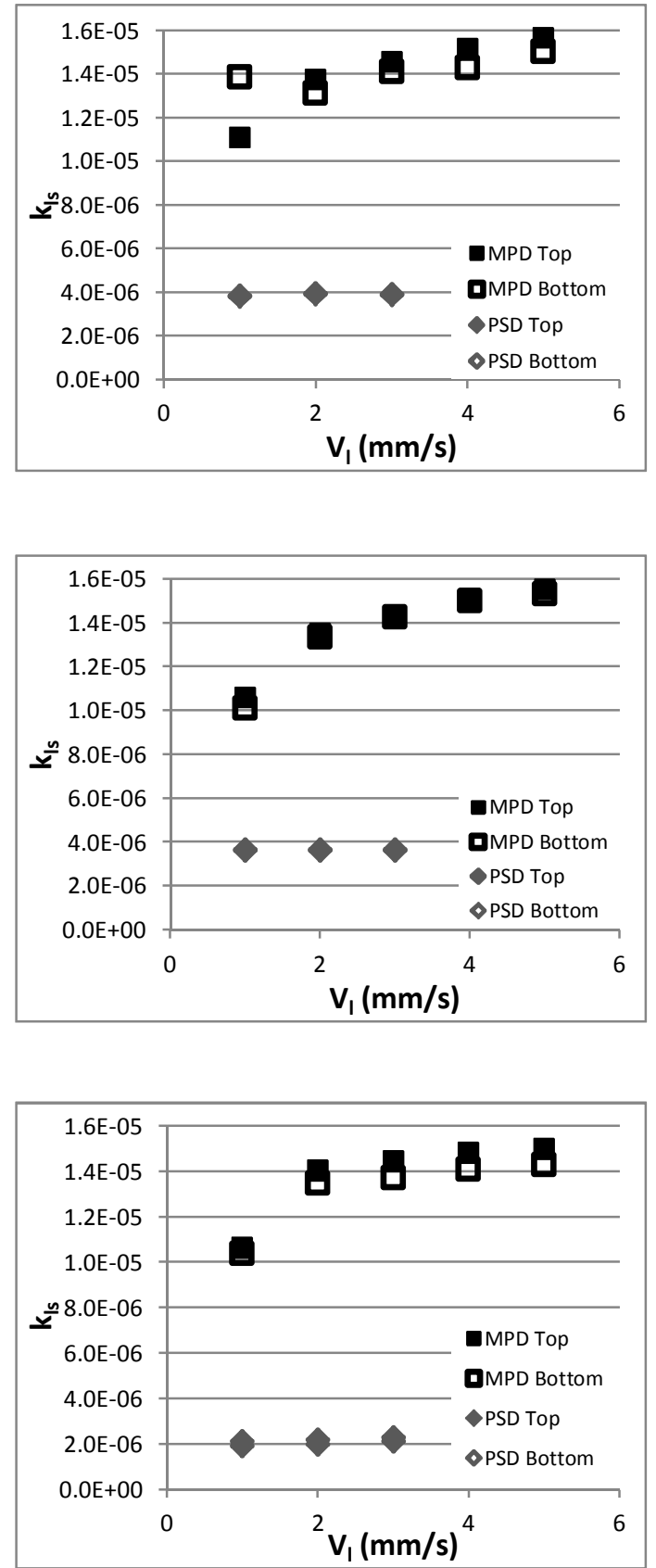

Figure 5: Specific mass transfer using a MPD and a PSD a)D/dp=3 b) $D / d p=6$ c) $D / d p=10$ 

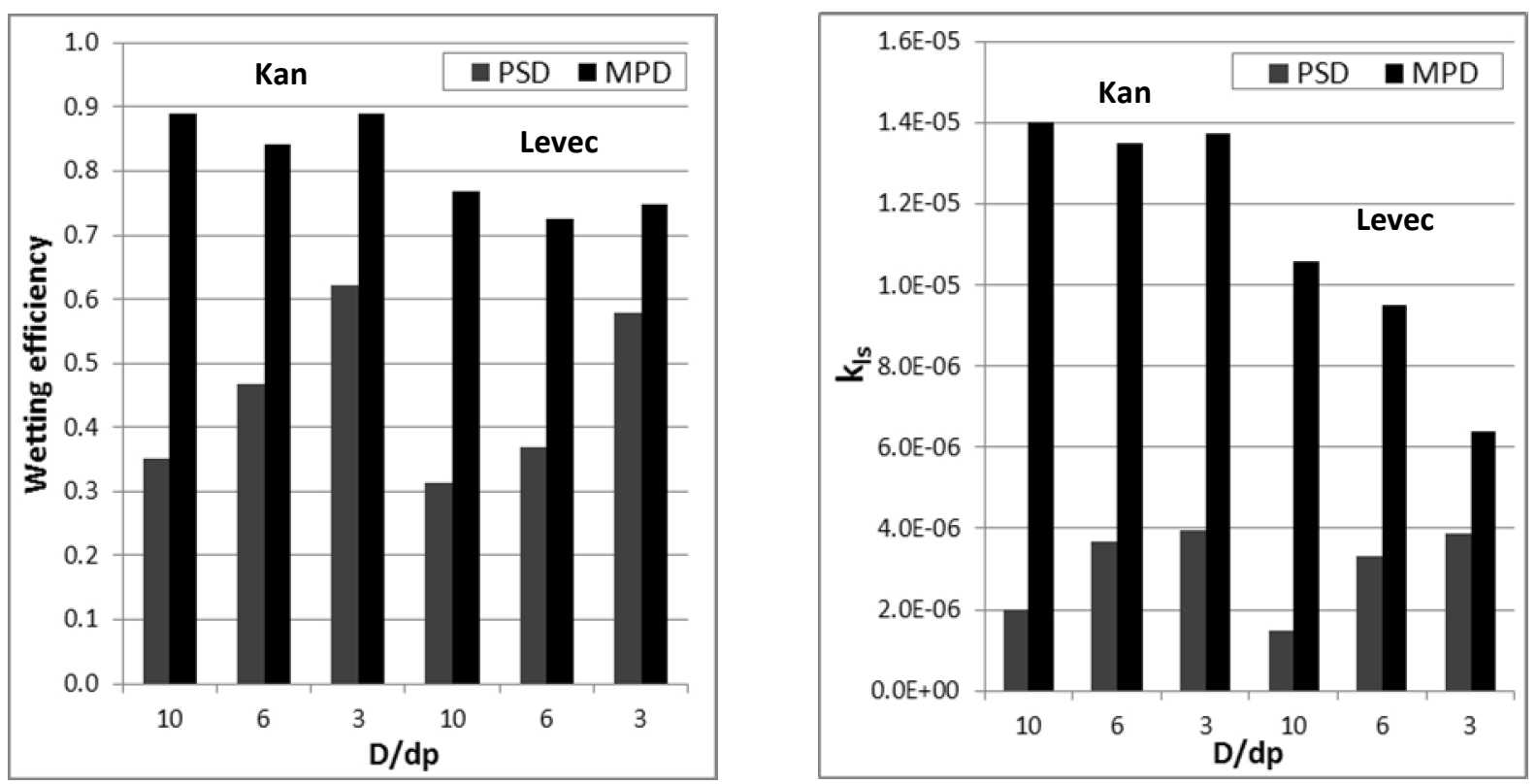

Figure 6: Experimental trends for PSD and MPD for the top electrode $\left(V_{l}=2 \mathrm{~mm} / \mathrm{s}, V_{g}=20 \mathrm{~mm} / \mathrm{s}\right)$

\section{Conclusions}

New ideas regarding the minimum column to particle ratio $(\mathrm{D} / \mathrm{dp})$ was introduced in this paper. It is suggested that proper prewetting and distribution can allow for representative reaction experiments at $\mathrm{D} / \mathrm{dp}$ values as low as 3 . It is preferable to test the postulate under real reaction conditions, but if true it opens new possibilities for laboratory scale representation of industrial reactors.

\section{Nomenclature}
$A \quad$ - Geometrically calculated electrode area $\left(\mathrm{m}^{2}\right)$
C - Concentration $\left(\mathrm{mol} / \mathrm{m}^{3}\right)$
Ilim $\quad$ - Limiting current (A)
$I_{\text {measured }} \quad$ - Current measured for wetting efficiency (A)
F - Faraday constant (c/mol)
kls - Specific mass transfer based on wetted area $(\mathrm{m} / \mathrm{s})$
$n \quad-$ Number of electrons involved in stoichiometric equation (-) 


\section{References}

Al-Dahhan, M.H. and M.P. Dudukovic, "Pressure drop end liquid hold-up in high pressure trickle bed reactors,” Chem Eng Sci. 49 , 5681-5698 (1994)

Attou, A. and C. Boyer, "Hydrodynamics of gas-liquid-solid trickle bed reactors: a Critical review," Oil \& Gas Science and Technology 54 , 29-66 (1999)

Baussaron, L., C. Julcour-Lebigue, A. Wilhelm, H. Delmas and C. Boyer, "Wetting topology in trickle bed reactors," AIChE J. 53 , 1850-1860 (2007)

Baker, T., T.H. Chilton and H.C. Vemon, "The course of liquid flow in packed towers," Trans. Amer. Inst. Chem. Eng. 31 , 296-306 (1935)

Benenati, R.F. and C.B. Brosilow, "Void fraction distribution in beds of spheres," AIChE J. $\mathbf{8}, 359-361(1962)$

De Klerk, A. , "Voidage variation in packed beds at small column to particle diameter ratio," AIChE J. 49 , 2022-2029 (2003)

Gianetto, A., G. Baldi and V. Specchia, "Absorption in packed towers with concurrent downward high-velocity flows," Quad Chem Ital. 6, 125-130 (1970)

Gierman, H., "Design of laboratory hydrotreating reactors scaling down of trickle-flow reactors," App. Cat. 43 , 277-286 (1988)

Hanratty, T.J. and J.A. Campbell, "Measurement of wall shear stress," in "Fluid mechanic measurements,” R.J. Goldstein, Hemisphere publishing Corporation (1983)

Joubert, R. and W. Nicol, "Novel method for simulateous measurement of trickle flo liquid solid mass transfer and wetting efficiency," Unpublished work (2011) 
Larachi, F., A. Laurent, N. Dudukovic and G. Wild, "Experimental study of a trickle bed reactor operating at high pressure: Two phase pressure drop and liquid saturation," Chem Eng Sci. 46 , 1233-1246 (1991)

Loudon, D., W. Van der Merwe and W. Nicol, "Multiple hydrodynamic states in trickle flow; Quantifying the extent of pressure drop, liquid holdup and gas-liquid mass transfer variation," Chem Eng Sci. 61 , 7551-7562 (2006)

Metha, D. and M.C. Hawley, "Wall effect in packed columns,"Ind. Eng. Chem. Process Des. Dev. $8,280-282$ (1969)

Mears, D.E., "Test for transport limitations in experimental catalytic reactors,” Ind. Eng. Chem. Process Des. Dev. 10, 541-547 (1971)

Raichura, R.C., "Variation of overall voidage with tube-to-particle diameter ratio" Taylor and Francis Group 12, 309-327 (1999)

Rao, V.G. and A.A.H. Drinkenburg, "Solid liquid mass transfer in packed beds with cocurrent gas-liquid downflow," AIChE J. 31 , 1059-1068 (1985)

Ren, X., S. Stapf and B. Blumich, "Magnetic resonance visualisation of flow and pore structure in packed beds with low aspect ratio," Chem Eng Technol. 28 , 219-225 (2005)

Sie, S.T. and R. Krishna, "Process development and scale up:III. Scale-up and scale-down of trickle bed processes” Reviews in Chemical Engineering 14, 203-252 (1998)

Templeman J.J. and K.E. Porter, "Experimental determination of wall flow in packed columns," Chem Eng Sci. 21 , 1139-1140 (1965)

Van der Merwe, W. and W. Nicol, "Cherecterization of multiple flow morphologies within the trickle flow regime," Ind. Eng. Chem. Res. 44, 9446-950 (2005) 
Van Houwelingen, A.J., C. Sandrock and W. Nicol, "Particle wetting distribution in trickle bed reactors," AIChE J. 52 , 3532-3542 (2006)

Winterberg, M. and E. Tsotsas, "Impact of tube-to-particle-diameter ratio on pressure drop in packed beds,” AIChE J. 46 , 1084-1088 (2000)

Wang , J., "Analytical electrochemistry," $3^{\text {rd }}$ Edition, John Wiley \& Sons Inc., New Jersey (2006) 